\title{
Propriedades Físicas da Madeira de Eucalyptus cloeziana F. Muell
}

\author{
Rejane Costa Alves ${ }^{1}$, Ana Lúcia C. Oliveira ${ }^{1}$, Edgar Vladimiro Mantilla Carrasco ${ }^{1}$ \\ ${ }^{1}$ Universidade Federal de Minas Gerais - UFMG, Belo Horizonte/MG, Brasil
}

\section{RESUMO}

Foram avaliadas propriedades físicas da madeira de 70 árvores da espécie Eucalyptus cloeziana com aproximadamente 10 anos de idade, provenientes de florestas localizadas na região centro-oeste do Estado de Minas Gerais. Foram determinados umidade, densidade básica e aparente e retratibilidade. As amostras apresentaram valores médios de $703 \mathrm{~kg} / \mathrm{m}^{3}, 843 \mathrm{~kg} / \mathrm{m}^{3} \mathrm{e}$ $916 \mathrm{~kg} / \mathrm{m}^{3}$ para densidade básica, densidade aparente seca e densidade aparente a $12 \%$ de umidade, respectivamente. Quanto à retração radial, tangencial e axial foram encontrados, respectivamente, valores médios de 7,08,11,16 e 0,26\%. A retração volumétrica média foi de 19,68\% e o coeficiente anisotrópico, de 1,60, dessa forma a madeira é considerada estável dimensionalmente.

Palavras-chave: Eucalyptus cloeziana, densidade, retratibilidade.

\section{Physical Properties of Eucalyptus cloeziana F. Muell}

\begin{abstract}
We analyzed the wood physical properties of 70 Eucalyptus cloeziana trees, approximately 10 years old, from forests located in the Midwestern region of Minas Gerais. Moisture, basic and apparent density and shrinkage were determined. The samples showed mean values of $703 \mathrm{~kg} / \mathrm{m}^{3}, 843 \mathrm{~kg} / \mathrm{m}^{3}$ and $916 \mathrm{~kg} / \mathrm{m}^{3}$ for basic density, dry bulk density and apparent density at $12 \%$ moisture, respectively. Concerning the radial, tangential and axial shrinkage were found with average values of $7.08 ; 11.16$ and $0.26 \%$, respectively. The average volume shrinkage was $19.68 \%$, and the anisotropic ratio of 1.60 , thus the timber is considered stable dimensionally.
\end{abstract}

Keywords: Eucalyptus cloeziana, density, retratibility. 


\section{INTRODUÇÃO}

Diversos trabalhos têm sido realizados em madeira de eucalipto com o intuito de caracterizar as propriedades desse material (Latorraca \& Albuquerque, 2000; Paes et al., 2001; Araújo et al., 2004; Wu et al., 2006; Cabral et al., 2006, 2007; Rodrigues et al., 2008; Trugilho, 2009). Essa madeira atualmente é utilizada como matéria-prima em vários setores, como os de mobiliário, construção civil, energia, celulose e papel, dentre outros, dessa forma vem se firmando industrialmente no Brasil.

Entre as características mais importantes das árvores quanto à qualidade da madeira, as propriedades físicas e mecânicas se destacam. A qualidade se refere à combinação das características físicas, mecânicas, químicas e anatômicas da árvore, as quais permitem a melhor utilização da madeira para um determinado uso (Gonçalez et al., 2006). A qualificação da madeira, segundo Rodrigues (2002), leva em consideração todos os fatores que alteram as características do material, como o comportamento físico-mecânico, que se sabe variável entre espécies e até mesmo entre madeiras.

Englerth (1966) menciona que a qualidade da madeira sólida é quase sinônimo de sua densidade e diversos autores atualmente utilizam essa propriedade física para explicar o comportamento físico-mecânico da madeira e qualificar sua utilização (Bortoletto, 2008; Parolin \& Worbes, 2000; Peltola et al., 2009; Wang et al., 2000; Repola, 2006). Outra característica fundamental para qualificar a madeira para determinada utilização é seu coeficiente anisotrópico (relação entre a contração tangencial e radial). Washusen et al. (2001), Silva \& Oliveira (2003), Pilura et al. (2005), Wang et al. (2008), Gonçalves et al. (2009), Silva et al. (2006) e Vidaurre et al. (2013) utilizam a densidade e o coeficiente de anisotropia para qualificar as madeiras quanto à estabilidade dimensional. Segundo Oliveira \& Silva (2003), esse coeficiente varia geralmente de 1,5 a 2,5 . É importante ressaltar que quanto menor essa relação, menos fendilhamento, rachaduras e empenamentos a madeira sofrerá.

Especificamente, a madeira de eucalipto cloeziana é pouco utilizada na construção civil, assim o objetivo do trabalho é a caracterização física e a determinação do coeficiente anisotrópico da madeira dessa espécie proveniente de florestas localizadas em regiões do Estado de Minas Gerais, mais precisamente nos municípios de Montes Claros e Bom Despacho.

\section{MATERIAL E MÉTODOS}

Foi definida uma grande quantidade de árvores com a finalidade de se obter o maior número possível de corpos de prova $(\mathrm{CP})$ válidos, visto que algumas madeiras de eucalipto apresentam fissuras logo após o corte, o que dificulta a retirada de CP que, segundo especificações da NBR 7190/1997 (ABNT, 1997), devem ser isentos de defeitos e retirados de regiões afastadas das extremidades das peças.

\subsection{Amostragem das toras na floresta e preparação dos lotes de madeira}

Foram coletadas amostras de 70 árvores da espécie Eucalyptus cloeziana, com idade em torno de 10 anos, da fazenda de propriedade da Cia. Agroflorestal de Santa Bárbara - CAF Santa Bárbara, localizada na região do município de Bom Despacho. Essa madeira é proveniente de clones, com espaçamento e tratos silviculturais compatíveis com a finalidade de uso da madeira para construção civil. Bom Despacho localiza-se a $19,7^{\circ}$ de latitude sul, altitude de $769 \mathrm{~m}$, clima tropical e temperatura média anual de $22,1^{\circ} \mathrm{C}$.

De cada árvore, acima dos 1,30 de altura, foram retirados quatro toretes de $100 \mathrm{~cm}$ de comprimento e de cada torete retiraram-se os CPs isentos de defeito para os ensaios de caracterização física.

\subsection{Acondicionamento dos toretes e preparação dos corpos de prova}

Os toretes ficaram acondicionados próximos à marcenaria em temperatura ambiente, sob cobertura e ventilação, durante 3 anos. Dessa forma, até o início dos ensaios, os toretes secaram ao ar, entrando em equilíbrio com o ambiente (equilíbrio higroscópico). A partir desses toretes foram preparados todos os CP para os ensaios de caracterização física da madeira, seguindo-se os procedimentos especificados pela NBR 7190/1997, anexo B. Os CP foram sempre retirados do cerne do torete. 


\subsection{Caracterização física}

Foram realizados ensaios para a determinação de umidade, densidade, retração e inchamento, seguindo procedimentos e métodos de ensaio da NBR 7190/1997. $\mathrm{O}$ teor de umidade da madeira (U em \%) corresponde à relação entre a massa de água nela contida e a massa da madeira seca, Equação 1.

$$
U(\%)=\frac{M_{i}-M_{s}}{M_{s}} \times 100
$$

em que: $\mathrm{Mi}=$ massa inicial da madeira, em gramas (g); $\mathrm{Ms}=$ massa da madeira seca, em gramas $(\mathrm{g})$.

A densidade básica é a massa específica convencional definida pela razão entre massa seca e volume saturado, Equação 2.

$$
\rho_{\text {bas }}=\frac{M_{s}}{V_{\text {sat }}}
$$

em que: $\mathrm{Ms}=$ massa da madeira seca, $\mathrm{em} \mathrm{kg}$; Vsat = volume da madeira saturada, $\mathrm{em}^{3}$.

A massa específica aparente a $12 \%\left(\rho_{\text {app }}, 12 \%\right)$ é definida pela razão entre massa e volume da madeira com teor de umidade de 12\%, Equação 3.

$\rho_{a p, 12 \%}=\frac{M_{12}}{V_{12}}$

em que: $\mathrm{M}_{12}=$ massa da madeira a $12 \%$ de umidade, em kg; Vsat = volume da madeira a $12 \%$ de umidade, em $\mathrm{m}^{3}$.

O valor das densidades: aparente na umidade do $\mathrm{CP},\left(\mathrm{D}_{\mathrm{ap}, \mathrm{n} \%}\right)$; aparente seca $\left(\mathrm{D}_{\mathrm{ap}, 0 \%}\right)$; e básica $\left(\mathrm{D}_{\mathrm{bas}}\right)$ foi determinado a partir dos resultados experimentais. A umidade dos CP variou de $10,45 \%$ a $39,16 \%$. A densidade aparente a $12 \%$ de umidade $\left(\mathrm{D}_{\text {ap. } .12 \%}\right)$ foi determinada utilizando-se a equação de correção apresentado pela ISO 3130/1975 (ISO, 1975), Equação 4. O número de amostras foi de 434 .

$$
\rho_{12}=\rho_{w} \cdot\left[1-\frac{(1-K) X((W-12)}{100}\right]
$$

em que: $\mathrm{w}=$ teor de umidade do $\mathrm{CP}$, $\mathrm{em} \% ; \rho_{\mathrm{W}}=$ densidade aparente a $\mathrm{w} \%$ de teor de umidade, em $\mathrm{kg} / \mathrm{m}^{3}$; $\mathrm{K}=$ coeficiente de retratibilidade volumétrica para mudança de $1 \%$ de umidade, igual a $0,8510^{-3} \mathrm{x} \rho_{\mathrm{w}}$ (valor obtido experimentalmente referente à amostra estudada).

\section{RESULTADOS E DISCUSSÃO}

Os resultados das densidades aparente e básica de ensaio e os calculados estão apresentados na Tabela 1 e na Figura 1.

Na Figura 1 pode-se observar que os resultados dos ensaios apresentam distribuição normal. Isso foi verificado pelo teste de normalidade Anderson-Darling ( $\mathrm{P}$ value $<0,3$ ), assim as estimativas da média, desvio-padrão e coeficiente de variação podem ser aceitos.

O valor médio da densidade básica e aparente a 12\% de umidade das madeiras de Eucalyptus cloeziana foi $703 \mathrm{~kg} / \mathrm{m}^{3}$ e $916 \mathrm{~kg} / \mathrm{m}^{3}$, respectivamente, o que indica que estão inseridas na classe C30 da NBR 7190/1997. Os valores encontrados na literatura foram semelhantes ao obtidos no presente trabalho. A NBR 7190/1997 e Calil et al. (2006) indicam um valor médio de densidade aparente a $12 \%$ de umidade de $820 \mathrm{~kg} / \mathrm{m}^{3}$ para a mesma espécie de madeira, enquanto Borges (2008) encontrou valor médio de densidade aparente de $790 \mathrm{~kg} / \mathrm{m}^{3}$ para árvores de 37 anos. Já Pereira et al. (2000) e Gonçalez et al. (2006) encontraram valores de densidade básica de $680 \mathrm{~kg} / \mathrm{m}^{3}$ e $670 \mathrm{~kg} / \mathrm{m}^{3}$ para o cloeziana com 6,5 e 17 anos, respectivamente.

Na Tabela 2 e na Figura 2, são apresentados os coeficientes de retratibilidade e coeficiente anisotrópico. Esse último coeficiente é definido pela relação entre a retração na direção tangencial dividida pela mesma propriedade na direção radial (R.T./R.R.).

Na Figura 2 se pode observar que os resultados apresentam distribuição normal. Isto foi verificado pelo

Tabela 1. Densidade aparente e densidade básica de Eucalyptus cloeziana.

Table 1. Apparent density and basic density of Eucalyptus cloeziana.

\begin{tabular}{cccccc} 
Parâmetros $\left(\mathbf{k g} / \mathbf{m}^{3}\right)$ & Média & D.P. & C.V. & Mínimo & Máximo \\
\hline $\mathrm{D}_{\text {bas }}$ & 703 & 71 & 0,11 & 520 & 899 \\
$\mathrm{D}_{\text {ap }, 0 \%}$ & 843 & 95 & 0,12 & 521 & 1071 \\
$\mathrm{D}_{\text {ap }, 12 \%}$ & 916 & 110 & 0,12 & 595 & 1189 \\
\hline
\end{tabular}



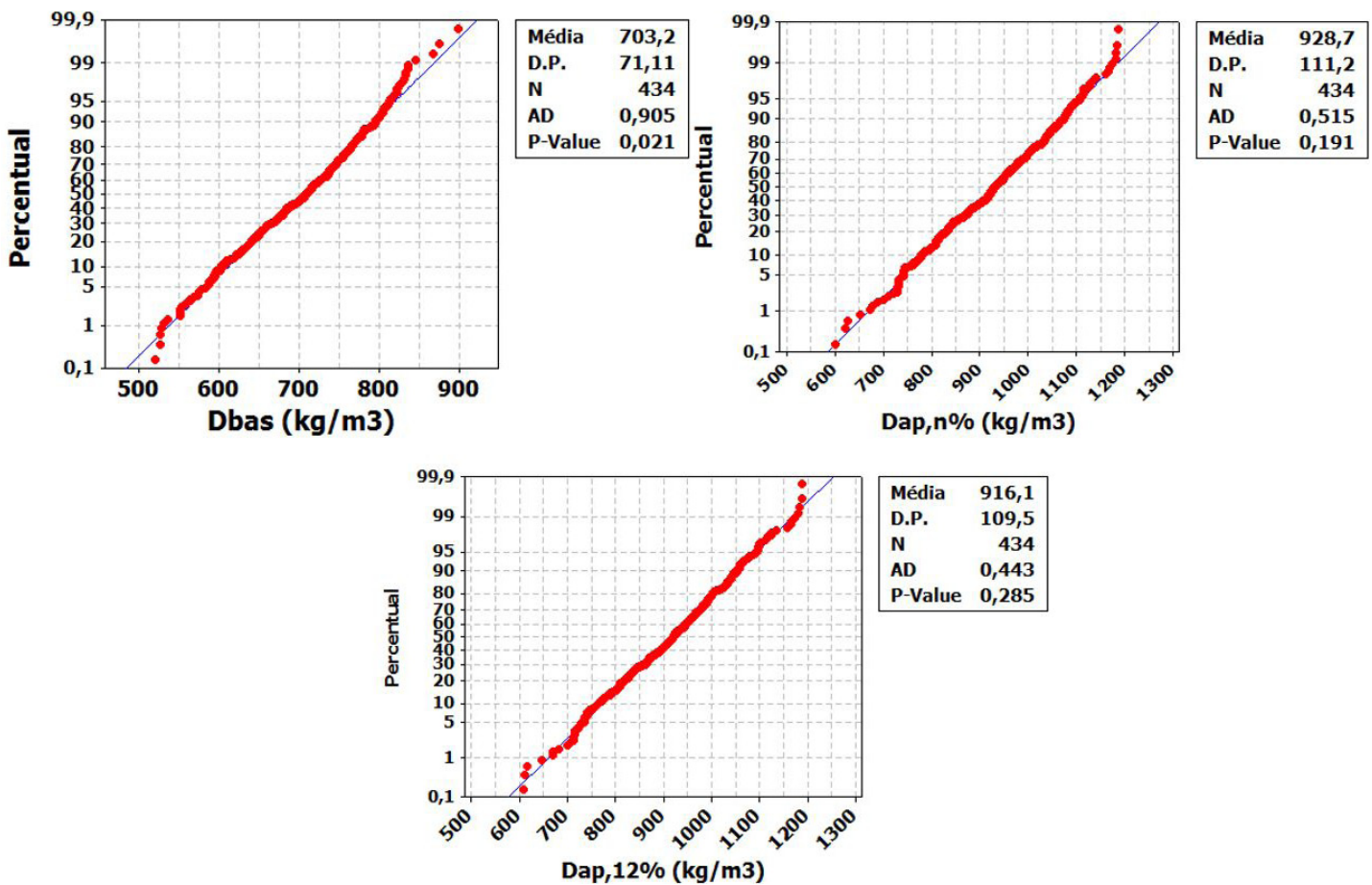

P-Value $\mathbf{0 , 2 8 5}$

Figura 1. Gráficos estatísticos das densidades.

Figure 1. Statistical graphs of density.

Tabela 2. Coeficiente anisotrópico da madeira de Eucalyptus cloeziana.

Table 2. Coeficient of Eucalyptus cloeziana anisotropic.

\begin{tabular}{|c|c|c|c|c|c|}
\hline \multirow{2}{*}{$\begin{array}{l}\text { Eucalyptus } \\
\text { cloeziana }\end{array}$} & \multicolumn{3}{|c|}{ Retratibilidade } & \multirow{2}{*}{$\begin{array}{l}\text { Coeficiente } \\
\text { anisotrópico }\end{array}$} & \multirow{2}{*}{$\begin{array}{c}\text { Variação } \\
\text { volumétrica (\%) }\end{array}$} \\
\hline & $\begin{array}{c}\text { Radial } \\
(\%)\end{array}$ & $\begin{array}{c}\text { Tangencial } \\
(\%)\end{array}$ & $\begin{array}{c}\text { Longitudinal } \\
(\%)\end{array}$ & & \\
\hline Média & 7,08 & 11,16 & 0,26 & 1,60 & 19,68 \\
\hline DP & 1,74 & 2,56 & 0,13 & 0,26 & 4,53 \\
\hline Mínimo & 2,53 & 4,58 & 0,04 & 1,01 & 10,02 \\
\hline Máximo & 11,89 & 18,50 & 0,56 & 2,16 & 29,93 \\
\hline
\end{tabular}

$\mathrm{DP}=$ desvio-padrão.

teste de normalidade Anderson-Darling (P value $<0,3$ ), assim as estimativas da média, desvio-padrão e coeficiente de variação podem ser aceitos.

Os valores médios encontrados para retração radial, tangencial e longitudinal das madeiras de Eucalyptus cloeziana foram próximos aos encontrados por outros pesquisadores (Trugilho et al., 1997; Lelles \& Silva, 1997; Gonçalez et al., 2006; Oliveira et al., 2010).

Coronel (1994) utiliza critérios para classificar a madeira com base no coeficiente anisotrópico. Valores entre 1,2 e 1,5 são considerados índices excelentes, já entre 1,5 e 2,0 são considerados normais ou médios e os maiores do que 2,0 são considerados ruins. As madeiras com esses últimos índices apresentam dificuldade para determinados usos devido a serem propensas ao fendilhamento, rachaduras e empenamentos. A madeira Eucalyptus Cloeziana enquadra-se na classificação normal ou média por apresentar coeficiente anisotrópico de 1,6. Durlo \& Marchiori (1992) confirmam que as madeiras de Eucalyptus cloeziana se enquadram nessa classificação, assim como Oliveira et al. (2010), que encontraram um valor de 1,7 para a mesma espécie de madeira. 

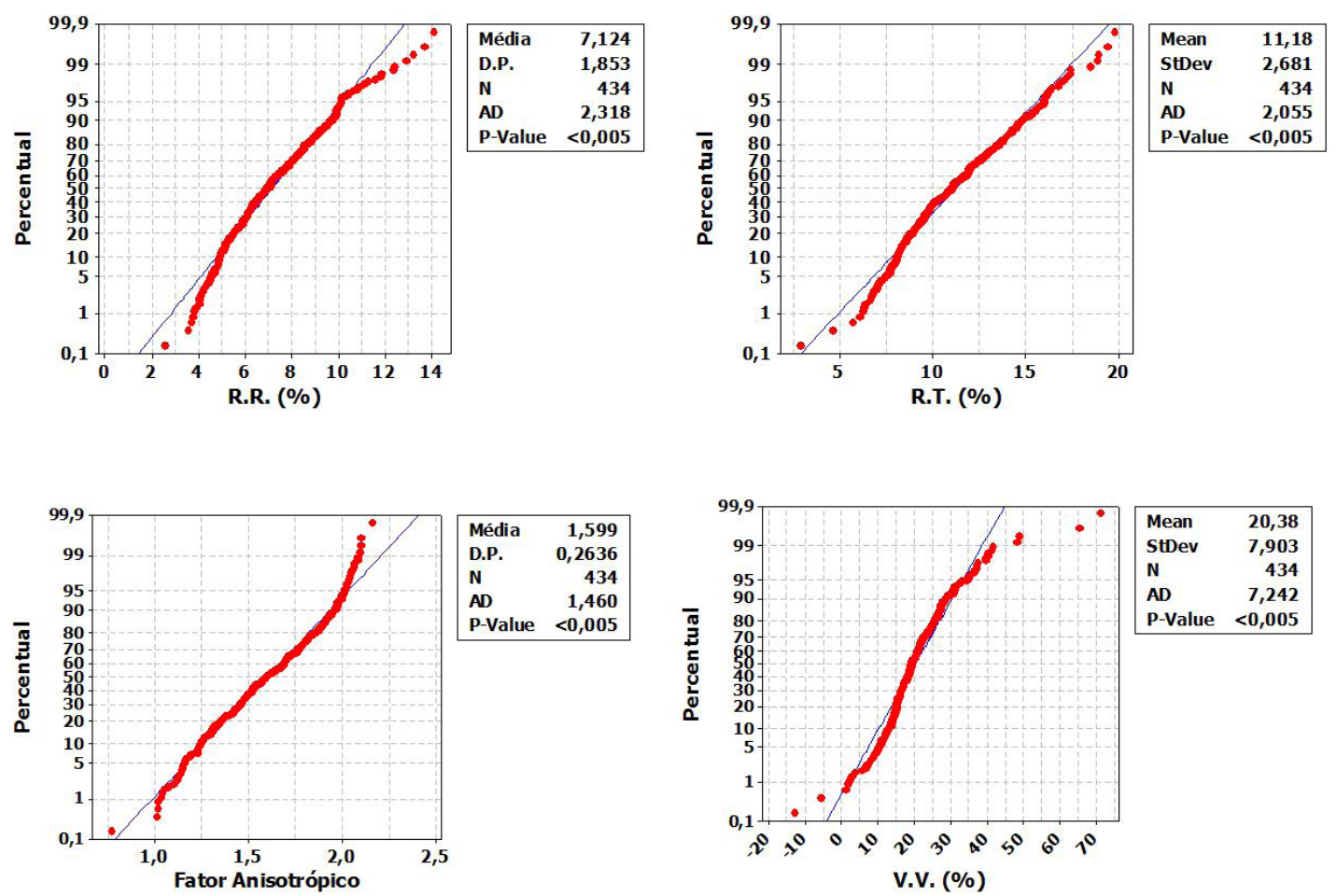

Figura 2. Gráficos estatísticos de retrações, coeficiente anisotrópico e variação volumétrica.

Figure 2. Statistical graphs of retractions, anisotropic factor and volumetric variation.

\section{CONCLUSÃO}

Com base nos resultados encontrados no presente trabalho conclui-se que as amostras de madeiras de Eucalyptus cloeziana apresentaram densidade com valores médios de: $703 \mathrm{~kg} / \mathrm{m}^{3} ; 843 \mathrm{~kg} / \mathrm{m}^{3} ; 929 \mathrm{~kg} / \mathrm{m}^{3}$; e $916 \mathrm{~kg} / \mathrm{m}^{3}$ para: densidade básica; densidade aparente seca; densidade aparente com umidade entre 10,45\% a $39,16 \%$; e densidade aparente a $12 \%$ de umidade, respectivamente. Quanto à retração radial, tangencial e axial, foram encontrados valores médios de 7,08, 11,16 e 0,26, respectivamente. A variação volumétrica foi de 19,68\% e o coeficiente anisotrópico, de 1,60, dessa forma, quanto à estabilidade dimensional, a madeira de Eucalyptus cloeziana é considerada estável.

\section{STATUS DA SUBMISSÃO}

Recebido: 19 set., 2014

Aceito: 5 abr., 2016

\section{AUTOR(ES) PARA CORRESPONDÊNCIA}

\section{Rejane Costa Alves}

Escola de Engenharia, Universidade Federal de Minas Gerais - UFMG, Avenida Antônio Carlos, 6627, Bloco 1, 4 andar, Pampulha, CEP 31270-901, Belo Horizonte, MG, Brasil e-mail: rejane.alves@ufersa.edu.br

\section{REFERENNCIAS}

Araújo CVM, Alves LJ, Santos OS, Alves JM. Micorriza arbuscular em plantações de Eucalyptus cloeziana F. Muell no litoral norte da Bahia, Brasil. Acta Botanica Brasílica 2004; 18(3): 513-520. http://dx.doi.org/10.1590/S010233062004000300011 .

Associação Brasileira de Normas Técnicas - ABNT. Projeto de estruturas de madeira: projeto NBR 7190/1997. Rio de Janeiro: ABNT, 1997. 107 p.

Borges CC. Potencialidade do uso de cruzetas de madeira tratada [dissertação]. Curitiba: Universidade Federal do Paraná; 2008. 
Bortoletto JG. Estudo de algumas propriedades físicas e mecânicas da madeira de Pinus merkusii. Revista Scientia Forestalis 2008; 36(79): 237-243.

Cabral CP, Vital BR, Della Lucia RM, Pimenta AS. Propriedades de chapas de aglomerado confeccionadas com misturas de partículas de Eucalyptus sp. e Pinus elliotti. Revista Árvore 2007; 31(5): 897-905. http://dx.doi. org/10.1590/S0100-67622007000500014.

Cabral CP, Vital BR, Della Lucia RM, Pimenta AS, Soares CP, Carvalho AMML. Propriedades de chapas tipo OSB, fabricadas com partículas acetiladas de madeiras de Eucalyptus grandis, Eucalyptus urophilla, Eucalyptus cloeziana e Pinus elliottii. Revista Árvore 2006; 30(9): 659668. http://dx.doi.org/10.1590/S0100-67622006000400020.

Calil C Jr, Dias AA, Góes JLN, Cheung AB, Stamato GC, Pigozzo JC et al. Manual de projeto e construção de pontes de madeira. São Carlos: Suprema; 2006.

Coronel, E. O. Fundamentos de las propiedades físicas y mecánicas de las maderas, $1^{\circ}$ parte, fundamentos de las propiedades físicas de las maderas. 1. ed. Santiago del Estero: Instituto de Tecnología de la Madera, Facultad de Ciencias Forestales, Universidad Nacional de Santiago del Estero; 1994.

Durlo MA, Marchiori JNC. Tecnologia da madeira: retratibilidade. Santa Maria: UFSM/CEPEF/FATEC; 1992. 33 p. Série Técnica, n. 10.

Englerth GH. Framework of qualitative relationship in wood utilization. Madison: Forest Products Laboratory; 1966, p. 16.

Gonçalez JC, Breda LC, Barros JFM, Macedo DG, Janin G, Costa AFC et al. Características tecnológicas das madeiras de Eucalyptus grandis W.Hill ex Maiden e Eucalyptus cloeziana F. Muell visando ao seu aproveitamento na indústria moveleira. Revista Ciência Florestal 2006; 16(3): 329-341. http://dx.doi.org/10.5902/198050981912.

Gonçalves FG, Oliveira JTS, Lucia RMD, Nappo ME, Sartório RC. Densidade básica e variação dimensional de um híbrido clonal de Eucalyptus urophylla $\times$ Eucalyptus grandis. Revista Árvore 2009; 33(2): 277-288. http://dx.doi. org/10.1590/S0100-67622009000200009.

International Organization for Standardization - ISO. ISO 3130: wood determination of moisture content for physical and mechanical tests. Switzerland: ISO; 1975.

Latorraca JV, Albuquerque CEC. Efeito de rápido crescimento sobre as propriedades da madeira. Revista Floresta e Ambiente 2000; 7(1): 279-291.

Lelles JG, Silva JC. Problemas e soluções sobre rachaduras de topo de madeiras de Eucalyptus spp. nas fases de desdobro e secagem. Informe Agropecuário 1997; 18(186): 62-69.

Oliveira JTS, Silva JC. Variação radial da retratibilidade e densidade básica da Madeira de Eucalyptus saligna Sm. Revista Árvore 2003; 27(3): 381-385. http://dx.doi. org/10.1590/S0100-67622003000300015.
Oliveira JTS, Tomazello M Fo, Fiedler NC. Avaliação da retratibilidade da madeira de sete species de Eucalyptus. Revista Árvore 2010; 34(5): 929-936. http://dx.doi. org/10.1590/S0100-67622010000500018.

Paes JB, Moreschi JC, Lelles JG. Tratamento preservativo de moirões de bracatinga (Mimosa scabrella BENTH.) e de Eucalyptus viminalis lab. pelo método de imersão prolongada. Cerne 2001; 7(2): 65-80.

Parolin P, Worbes M. Wood density of trees in black water floodplains of rio Jaú national park, Amazonia, Brazil. Revista Acta Amazonica 2000; 30(3): 441-448. http:// dx.doi.org/10.1590/1809-43922000303448.

Peltola H, Gort J, Pulkkinen P, Zubizarreta Gerendiain A, Karppinen J, Ikonen V-P. Differences in growth and wood density traits in Scots Pine (Pinus sylvestris L.) genetic entries grown at different spacing and sites. Silva Fennica 2009; 43(3): 339-354. http://dx.doi.org/10.14214/sf.192.

Pereira JCD, Sturion JA, Higa AR, Higa RCV, Shimizu JY. Características da madeira de algumas espécies de eucalipto plantadas no Brasil. Colombo: Embrapa Florestas; 2000. 113 p. Embrapa Florestas Documentos, n. 38.

Pilura A, Yu Q, Zhang SY. Variation in wood density and shrinkage and their relationship to growth of selected young poplar hybrid crosses. Forest Science 2005; 5: 472-482.

Repola J. Models for vertical wood density of Scots pine, Norway spruce and birch stems, and their application to determine average wood density. Silva Fennica 2006; 40(4): 673-685. http://dx.doi.org/10.14214/sf.322.

Rodrigues EAC, Rosado SCS, Trugilho PF, Santos AM. Seleção de Clones de Eucalyptus as propriedades físicas da madeira 147 avaliadas em árvores no campo. Cerne 2008; 14(2): 147-152.

Rodrigues RAD. Variabilidade de propriedades físicomecânicas em lotes de madeira serrada de eucalipto para a construção civil [dissertação]. Piracicaba: Escola Superior de Agricultura Luiz de Queiroz; 2002.

Silva JC, Oliveira JTS. Avaliação das propriedades higroscópicas da madeira de Eucalyptus salignaSm., em diferentes condições de umidade relativa do ar. Revista Árvore 2003; 27(2): 233-239. http://dx.doi.org/10.1590/ S0100-67622003000200012.

Silva JC, Oliveira JTS, Xavier BA, Castro VR. Variação da retratibilidade da madeira de Eucalyptus grandis Hill Ex. Maiden, em função da idade e da posição radial no tronco. Revista Árvore 2006; 30(5): 803-810. http://dx.doi. org/10.1590/S0100-67622006000500014.

Trugilho PF. Densidade básica e estimativa de massa seca e de lignina na madeira em espécies de Eucalyptus. Ciência e Agrotecnologia 2009; 33(5): 1228-1239. http:// dx.doi.org/10.1590/S1413-70542009000500005.

Trugilho PF, Mendes LM, Silva JRM, Lima JT. Influência da idade nas características físicas, químicas e anatômicas da madeira de Eucalyptus grandis W. Hill ex Maiden. 
In: Proceedings of the IUFRO Conference on Silviculture and Improvement of Eucalypt; 1997; Salvador. Colombo: Embrapa; 1997. p. 269-275.

Vidaurre G, Lombardi LR, Nutto L, Oliveira JTS, Arantes MDC. Propriedades da Madeira de Reação. Floresta e Ambiente 2013; 20(1): 26-37. http://dx.doi.org/10.4322/ floram.2012.041.

Wang E, Chen T, Pang S, Karalus A. Variation in anisotropic shrinkage of plantationgrown Pinus radiata wood. Maderas. Ciencia y Tecnología 2008; 10(3): 243-249.

Wang T, Aitken N, Rozenberg B, Millie F. Selection for improved growth and wood density in Lodgepole pine: effects on radial patterns of wood variation. Wood and Fiber Science 2000; 32(4): 391-403.

Washusen R, Ades P, Evans R, Ilic J, Vinden P. Relationships between density, shrinkage, extractives content and microfibril angle in tension wood from three provenances of 10-year-old Eucalyptus globulusLabill. Holzforschung 2001; 55(2) http://dx.doi.org/10.1515/HF.2001.029.

Wu Y-Q, Hayashi K, Liu Y, Cai Y, Sugimori M. Relationships of anatomical characteristics versus shrinkage and collapse properties in plantation-grown eucalypt wood from China. Journal of Wood Science 2006; 52(3): 187-194. http://dx.doi.org/10.1007/s10086-005-0751-6. 